\title{
Trombosis senovenosa cerebral en un recién nacido con mutación MTHFR C677T tratado con enoxaparina
}

\section{Cerebral sinovenous thrombosis in a newborn with mutation of MTHFR C677T treated with enoxaparin}

\author{
Juan Fasce ${ }^{\mathrm{a}, \mathrm{b}, \mathrm{c}}$, Marcela Calbacho ${ }^{\mathrm{a}}$, María Oyarzun ${ }^{\mathrm{a}, \mathrm{b}}$, \\ Katya Reinbach ${ }^{\mathrm{a}, \mathrm{b}}$, Ariadna Daza ${ }^{\mathrm{a}, \mathrm{d}}$, Alfredo García-Alix ${ }^{\mathrm{c}, \mathrm{e}, \mathrm{f}}$
}

\author{
aHospital Clínico Regional Guillermo Grant Benavente, Concepción, Chile \\ bUniversidad de Concepción, Chile \\ cFundación NeNe, España \\ 'Becada. Programa de Especialista en Pediatría, Universidad de Concepción, Chile \\ eInstitut de Recerca Pediatrica Sant Joan de Dèu. Hospital Sant Joan de Déu. Universidad de Barcelona, España \\ ${ }^{\text {fC}}$ entro de Investigación Biomédica en Red de Enfermedades Raras, Madrid, España
}

Recibido: 12 de agosto de 2019; Aceptado: 28 de noviembre de 2019

¿Qué se sabe del tema que trata este estudio?

La trombosis senovenosa cerebral neonatal (TSVC), es una patología rara y generalmente grave por lo que se debe efectuar un diagnóstico precoz. Se conoce poco acerca de su patogenia por lo que su manejo es controversial.
¿Qué aporta este estudio a lo ya conocido?

En nuestro paciente el tratamiento con heparina de bajo peso molecular fue exitoso. La segunda RM a los dos meses mostró una adecuada repermeabilización y lo más importante no había acontecido ninguna extensión de la trombosis.

\section{Resumen}

La trombosis senovenosa cerebral neonatal (TSVC), es una patología rara y generalmente grave, de la cual se conoce poco sobre los mecanismos fisiopatológicos responsables y, aunque controvertido, se ha sugerido que la trombofilia genética, puede desempeñar un rol en la patogénesis. Debido a los temores de un sangrado intracraneal el tratamiento anticoagulante con heparina de bajo peso molecular es controvertido. Objetivo: presentar un recién nacido con una trombosis senovenosa cerebral neonatal, discutir los factores de riesgo trombofílico, y el manejo con heparina de bajo peso molecular de la trombosis venosa cerebral. Caso Clínico: Recién nacido de término que debutó a los 8 días de vida con convulsiones clónicas, rechazo al pecho más hipoactividad motora. La neuroimagen con RM mostró una TSVC involucrando múltiples senos venosos, un infarto hemorrágico talámico derecho y congestión venosa de la sustancia blanca frontal. El estudio de trombofilia puso de relieve una mutación homocigota del gen MTHFR C677T. El tratamiento con heparina de bajo peso molecular
Palabras clave: Convulsiones neonatales; trombosis senovenosa cerebral; mutación MTHFR C677T; infarto cerebral; anticoagulación

Correspondencia:

Juan Fasce

jafascec@yahoo.com 
se asoció a repermeabilización del seno sagital superior a los 23 días de iniciada la terapia. Conclusiones: La presentación clínica de la TSVC en el neonato es inespecífica, probablemente en relación con la extensión y gravedad de la lesión y el desarrollo de complicaciones asociadas, como infartos hemorrágicos venosos intraparenquimatosos o hemorragia intraventricular. Estas complicaciones son detectables mediante Ecografia o Resonancia Magnética, y deben hacer sospechar una TSVC. En esta experiencia el tratamiento anticoagulante mostró ser seguro y prevenir la extensión de la trombosis.

\section{Abstract}

Introduction: Neonatal cerebral sinovenous thrombosis (CSNT) is a rare and generally serious condition about which there is little knowledge of the responsible pathophysiological mechanisms and, although controversial, it has been suggested that genetic thrombophilia may play a role in its pathogenesis. Out of concern for intracranial bleeding, the anticoagulant treatment with low-molecularweight heparin is controversial. Objective: To present a case of a newborn with neonatal CSNT, to analyze the thrombophilic risk factors, and the management of cerebral venous thrombosis with low-molecular-weight heparin. Clinical Case: Full-term newborn who presented at eight days of life breastfeeding rejection, clonic seizures, and locomotor hypoactivity. The MRI neuroimaging showed a CSNT involving multiple venous sinuses, a right thalamic hemorrhagic infarction, and venous congestion in frontal white matter. Thrombophilia study highlighted a homozygous MTHFR C677T mutation. Treatment with low-molecular-weight heparin was associated with repermeabilization of the superior sagittal sinus after 23 days of starting therapy. Conclusions: The clinical presentation of CSNT in the neonate is nonspecific, probably related to the extent and severity of the injury and the development of associated complications, such as venous hemorrhagic infarctions and intraparenchymal or intraventricular hemorrhage. These complications are detected through ultrasound or MRI, and they should make us suspect a CSNT. In this experience, the anticoagulant treatment proved to be safe and prevents thrombus propagation.

\section{Keywords:}

Neonatal seizures; cerebral sinovenous thrombosis; MTHFR C677T mutation; stroke; anticoagulation

\section{Introducción}

La trombosis senovenosa cerebral neonatal (TSVC), es una patología rara, con una incidencia estimada entre 1 a 12 por 100.000 nacidos vivos año. Una incidencia superior a la referida en la edad pediátrica $(0,35-0,67 \text { por } 100.000 \text { niños/año })^{1-3}$. Aun así, es probable que el problema en neonatos se subestime, ya que la presentación clínica es inespecífica. De hecho, el diagnóstico de esta entidad está aumentando debido a un mayor índice de sospecha y la mayor disponibidad y capacidad diagnóstica de las técnicas de neuroimagen cerebral $^{1}$.

Aunque se han asociado varios factores de riesgo con la TSVC neonatal, se sabe poco acerca de los mecanismos fisiopatológicos responsables de la mayoría de los casos y se ha sugerido que la trombofilia genética (predisposición a la trombosis), puede desempeñar un rol en la patogénesis, particularmente las mutaciones del factor V Leiden (FV G1691A), la protrombina G20210A (FII G20210A) y la del MTHFR (C677T), ya que en adultos estos factores se han asociado con la TSVC. Sin embargo, la evidencia en las edades pediátricas y neonatales sigue siendo controvertida. ${ }^{4}$

Debido a la alta prevalencia de sangrado intracraneal en el recién nacido con TSVC, el tratamiento anticoagulante con heparina de bajo peso molecular es controvertido. No obstante, debido al riesgo de extensión del trombo y a que casi un 50\% de los neonatos con TSVC presentan discapacidad neurológica ulterior, el tratamiento anticoagulante se ha ido incorporando a pesar de una limitada experiencia.

El objetivo es presentar un recién nacido con una trombosis senovenosa cerebral neonatal, discutir los factores de riesgo trombofílico, y el manejo con heparina de bajo peso molecular de la trombosis venosa cerebral.

\section{Caso Clínico}

Neonato de sexo femenino que ingresa al octavo día de vida por presentar hipoactividad motora, rechazo del pecho y convulsiones clónicas en extremidades superiores asociadas a alteración en la mirada. Madre multípara, sin antecedentes mórbidos. Nació por parto vaginal eutócico a las 40 semanas de gestación, con un peso de 3660g. El Apgar fue de 9-9, al minuto y cinco minutos, respectivamente. Tras ingresar en la unidad de cuidado intensivo neonatal, se apreció estado convulsivo electroclínico mediante aEEG (electroencefalograma de amplitud integrada) (figura 1), iniciándose tratamiento con fenobarbital. Debido a la falta de respuesta a dicho fármaco se añadió fenitoína y ante el riesgo de depresión respiratoria, se intubó y se inició 


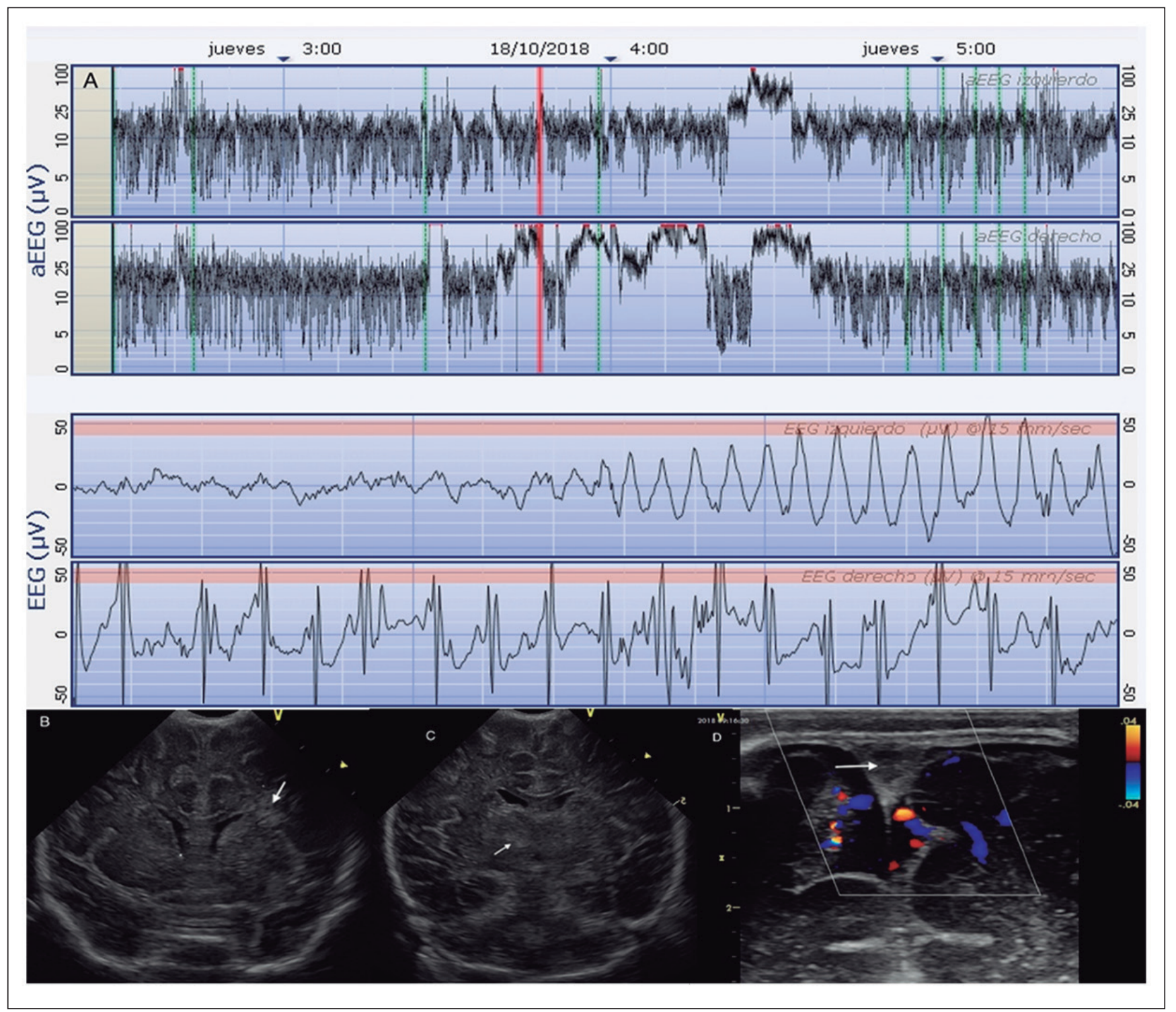

Figura 1. A. aEEG en las tres primeras horas de ingreso. Se aprecia estado epiléptico en el hemisferio derecho y convulsiones recurrentes en el hemisferio izquierdo. Las crisis comienzan en el lado derecho. B. Ultrasonografía cerebral (USC): Plano coronal 2, donde se aprecia ecogenicidad periventricular en el ángulo externo de los ventrículos laterales en forma de rayos de rueda, sugestiva de congestión de venas medulares (flecha). C. Plano coronal 3 donde se aprecia área hiperecogénica en el tálamo derecho (flecha fina) en área mesial, sugestiva de lesión hemorrágica-isquémica. D. USC-Doppler flujo color mostrando ausencia de flujo venoso en el SSS (flecha).

ventilación mecánica más tratamiento con ampicilina y cefotaxima, los cuales se suspendieron $48 \mathrm{~h}$ más tarde tras disponer de los hemocultivos, y Aciclovir que se suspendió a los 4 días del ingreso. La PCR de virus herpes (tipos 1, 2 y 6) fue negativa, la concentración de procalcitonina normal, y el LCR fue xantocrómico pero no presentó pleocitosis ni glucorraquia.

Una tomografía axial computarizada (TAC) cerebral al ingreso mostró trombosis venosa del seno sagital superior (SSS) e hipointensidad de la sustancia blanca frontal. La ecografía cerebral de ese mismo día mostró un área de ecogenicidad aumentada en el lado medial del tálamo derecho, pero no mostró imagen sugestiva de hemorragia intraventricular y si de congestión de los plexos coroideos (figura 1). El estudio de RM (secuencias T1W, T2W, DWC, ADC, SWI y TOF) confirmó la TSVC involucrando al seno sagital superior, al seno recto y la vena de galeno. Además, puso de manifiesto un infarto talámico derecho de carácter hemorrágico con un borde isquémico en el aspecto mesial del mismo. Además, presentaba congestión de las venas medulares en la sustancia blanca periventricular frontal (figura 2).

Tras $48 \mathrm{~h}$ sin nuevos episodios convulsivos, a los 
Figura 2. A-B. imágenes TW1 y TW2 RM mostrando lesión hemorrágica-isquémica del tálamo derecho. Además, se aprecia trombosis del SSS, así como congestión de las venas medulares en la sustancia blanca periventricular frontal. C. Imagen sagital media T2W que muestra trombosis del SSS, del seno recto (SR) y de la vena de Galeno (VG), y de las venas cerebrales internas (VCI). D. Imagen TOF mostrando ausencia de flujo venoso en seno recto y marcada reducción en el SSS.
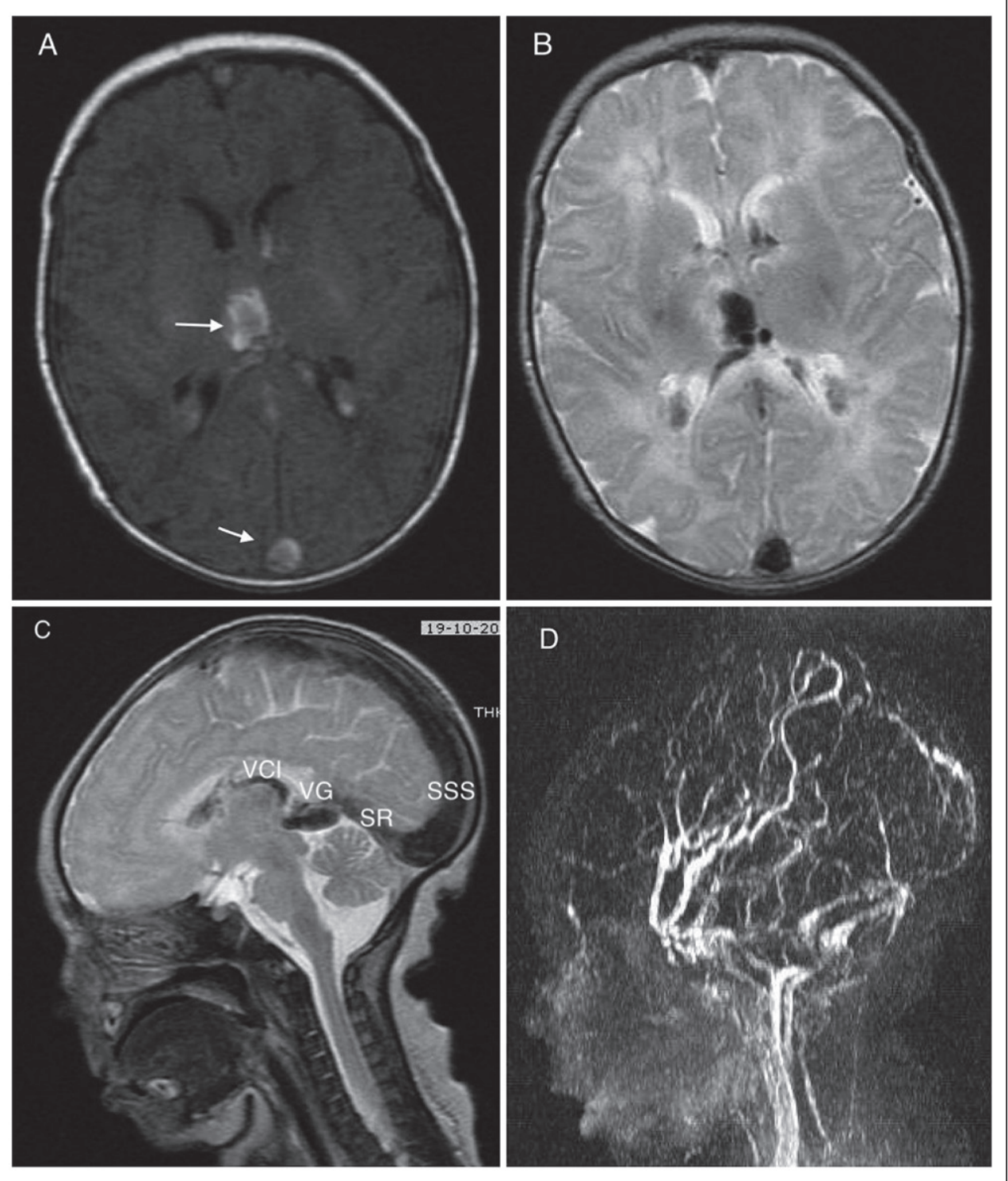

10 días de vida se retiró el soporte ventilatorio y la monitorización con el aEEG. El estudio de trombofilia (FV G1691A, protrombina G20210A, MTHFR (C677T), Antitrombina III, Proteínas C, S y homocisteina) no mostró alteraciones, excepto que el paciente era homocigoto para la mutación del MTHFR C677T. Se inició tratamiento con heparina de bajo peso molecular (enoxaparina): $1,5 \mathrm{mg} / \mathrm{kg} /$ dosis, una cada $12 \mathrm{~h}$ por vía subcutánea a los 12 días de vida. El día 35 de vida, tras 23 días con tratamiento anticoagulante, el eco-doppler cerebral mostró presencia de flujo venoso en seno sagital superior (figura 3). El paciente se da de alta a los 36 días de vida sin convulsiones, con buena succión y tratamiento con levetiracetam.
A los 2 meses de tratamiento anticoagulante, una angio-RM de control mostró repermeabilización de senos venosos, adelgazamiento del cuerpo calloso, reducción del volumen de la sustancia blanca y aumento del espacio extraxial (figura 3 ).

\section{Discusión}

La presentación clínica de la TSVC en el neonato es inespecífica y además muy variable, en expresión y gravedad, probablemente en relación con la extensión y magnitud de la obstrucción del drenaje venoso y el desarrollo de complicaciones asociadas, como infartos hemorrágicos venosos intraparenquimatosos o hemo- 

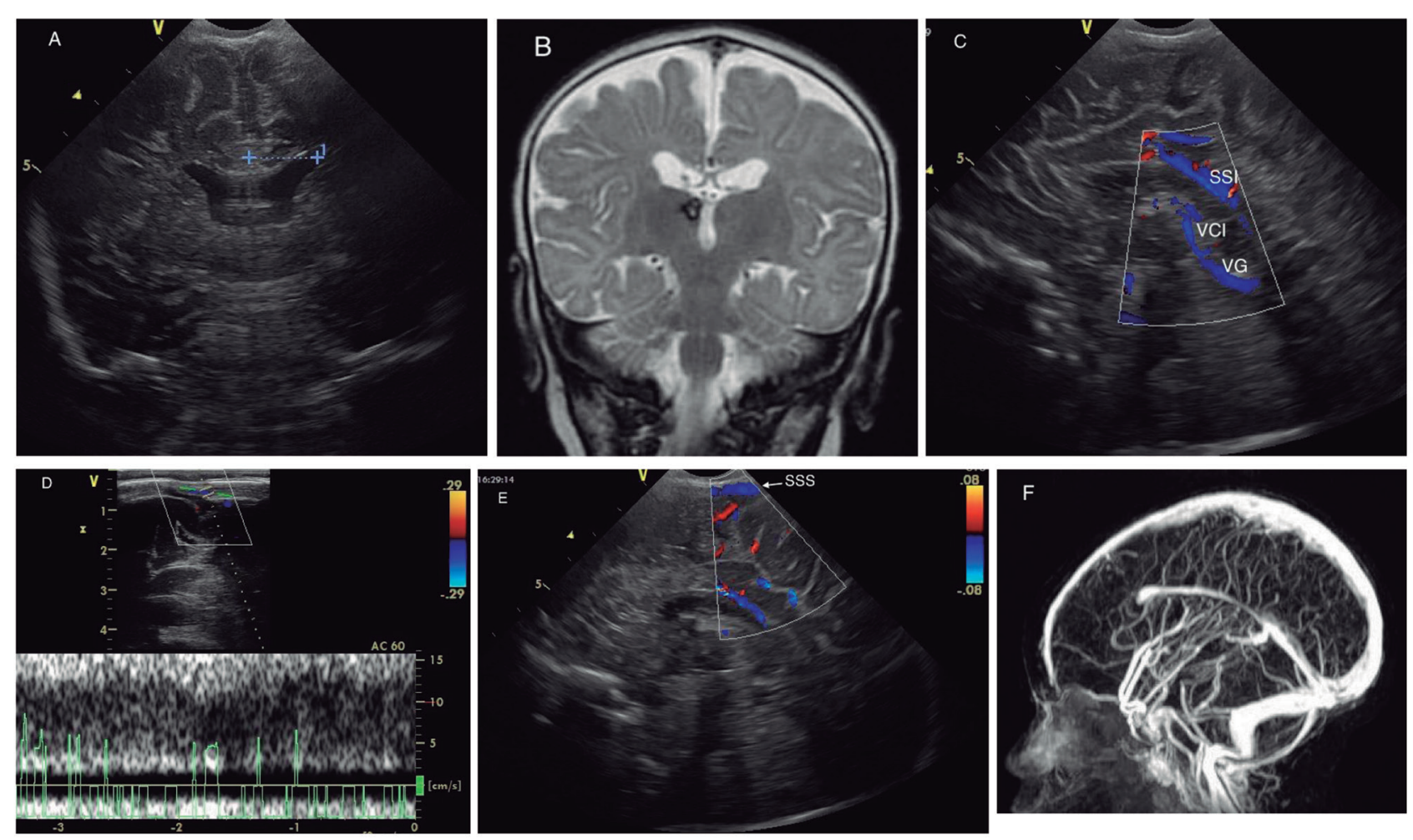

Figura 3. Estudio posterior al tratamiento anticoagulante. A. USC (35 días de vida). B. RM (dos meses de edad). A y B planos coronales a nivel del foramen de Monro, se aprecia ventriculomegalia, aumento del espacio extraaxial, cuerpo calloso adelgazado y reducción del volumen de la sustancia blanca. C. Doppler flujo color en sección sagital. Se aprecia flujo en los senos sagital inferior (SSI), venas cerebrales internas (VCI), y vena de Galeno (VG). D y E. Estudio Doppler mostrando flujo en el SSS. F. Estudio angiográfico de RM (TOF) mostrando un flujo adecuado en SSS, en el seno recto y la vena de Galeno.

rragia intraventricular. La expresión clínica cae dentro de tres categorías: a) convulsiones clónicas focales que resultan de un infarto venoso local o de una hemorragia; b) alteración cerebral difusa caracterizada por una combinación variable de convulsiones multifocales, disminución de la ingesta oral, irritabilidad, hipotonía, apnea (generalmente epiléptica) y/o deterioro de la conciencia (letargia, estupor o coma). Estas manifestaciones son posiblemente secundarias al aumento de la presión intracraneal debido a la obstrucción del drenaje venoso; c) como hallazgo ocasional, al realizar un estudio de imagen cerebral en un neonato hospitalizado por otra causa ${ }^{1,2,4}$. Mientras en algunas series las convulsiones son el signo de debut predominante, ello no es consistente en todos los estudios ${ }^{1,4-6}$. No obstante, como ilustra el paciente presentado, ante la presencia de convulsiones neonatales sean focales o multifocales se ha de considerar una posible trombosis senovenosa cerebral. A diferencia del infarto arterial isquémico, no es excepcional que el deterioro de la conciencia en estos pacientes forme parte del síndrome clínico.

La TSVC se puede demostrar mediante ecografía Doppler o estudios de imagen con RM incluyendo an- giografía de RM y particularmente venografía de $\mathrm{RM}^{5}$. La TAC permite también establecer el diagnóstico y aunque utilizada en nuestro paciente, no debería utilizarse en el neonato si hay disponibilidad de otras pruebas por su alta dosis de radiación ionizante. Aunque la Ecografia cerebral con Doppler color es un estudio muy específico para descartar TSVC y cuando se aprecia ausencia de flujo sugiere el diagnóstico, la RM es el estudio de elección, y la venografía de RM es hoy el estudio "gold standard" para establecer certeramente el diagnóstico ${ }^{4}$. La TSVC en nuestro paciente fue múltiple e involucró a los senos sagital superior y recto, los senos con más frecuencia afectados ${ }^{1,4}$. Entre las lesiones asociadas $(\approx 50 \%)$ en la TSVC, particularmente en la trombosis del seno recto, destaca la hemorragia talámica unilateral, la hemorragia intraventricular o el infarto hemorrágico parenquimatoso ${ }^{5,7}$. Nuestro paciente presentó la primera junto con otro hallazgo observado con frecuencia: la congestión de las venas medulares particularmente en la sustancia blanca periventricular frontal (figuras 1 y 2$)^{7}$. La presencia de hemorragia ventricular asociada con hemorragia talámica unilateral, lesiones fácilmente detectadas mediante 
USC, nos obligan a descartar una TSVC ${ }^{7}$. Además en nuestro caso fue apoyado el diagnóstico por un aEEG asimétrico con crisis epilépticas predominantes en el lado derecho ${ }^{8}$.

Una gran diversidad de factores maternos, perinatales y neonatales pueden contribuir al desarrollo de la TSVC ${ }^{1,4}$. Sin embargo, el único factor de riesgo potencial en el paciente presentado fue la mutación en homocigosis del MTHFR C677T. Este gen es responsable de producir la enzima metilentetrahidrofolato reductasa (MTHFR). Este enzima transforma los 5,10 metilentetrahidrofolatos en 5 metiltetrahidrofolatos. El polimorfismo 677T del MTHFR es termolábil, y cuando ambos alelos (homocigoto) están mutados comporta riesgo de enfermedad trombótica en edad temprana ${ }^{9}$. Aunque se han publicado casos aislados, así como estudios sin cohorte control, en la que varios pacientes con TVSC tenían mutación del MTHFR ${ }^{1,10}$, en un estudio que comparó la trombofilia genética entre 25 neonatos con TSVC y sus madres frente a 85 pares madre-hijo controles, no se encontró que la mutación MTHFR C677T fuese un factor de riesgo ${ }^{4}$. El único factor protrombótico observado fue el FII G20210A, el cual mostró un OR de 6,70 (95\% IC:0,65-69.22). En base a este estudio, no es posible estar seguro del papel de la mutación MTHFR C677T en la génesis de la TSVC en el paciente presentado. No obstante, hasta que haya más estudios, todos los neonatos con TSVC deberían ser estudiados de trombofilia, incluyendo al menos: FVG169A, FII G20210A y MTHFR C677T y cualquiera de estas mutaciones consideradas potenciales factores causales.

El tratamiento anticoagulante es recomendado en la TSVC del adulto y en el presente, también en el niño no lactante ${ }^{11}$. El tratamiento anticoagulante en el neonato es controvertido. Por un lado, existe la posibilidad de que en caso de no tratamiento la trombosis se extienda y, por otro lado, el temor a que en caso de tratar empeore la hemorragia intracraneal (HIC), tan frecuente en esta patología. Un reciente metanálisis que examinó la influencia de una HIC en la TSVC en neonatos tratados versus no tratados, no observó que el tratamiento anticoagulante se asociase con mayor mortalidad prealta, ni con complicaciones hemorrágicas. Por el contrario, la terapia anticoagulante redujo el riesgo de propagación de la trombosis ${ }^{12}$. Aunque la evidencia del beneficio del tratamiento es escasa y pro- cede de registros internacionales y no de estudios específicamente dirigidos a responder a la necesidad o no de terapia anticoagulante ni la eficacia de la misma, los datos disponibles sugieren que la anticoagulación es segura y previene la extensión de la TSVC ${ }^{13}$. En nuestro paciente, la segunda RM a los dos meses mostró una adecuada repermeabilización y lo más importante no había acontecido ninguna extensión de la trombosis.

\section{Conclusiones}

La trombosis senovenosa cerebral neonatal a pesar de ser una patología rara es frecuentemente grave, que exige un diagnóstico precoz y un plan de manejo adecuado. Frente a un neonato de término que presente convulsiones y no tenga causa precisa debemos completar el estudio de imágenes mediante USC y RM. La presencia de hemorragia intraventricular o hemorragia talámica obliga a descartar TSVC. El tratamiento anticoagulante, aunque controversial en neonatos, ha demostrado ser seguro y prevenir la extensión de la trombosis.

\section{Responsabilidades Éticas}

Protección de personas y animales: Los autores declaran que los procedimientos seguidos se conformaron a las normas éticas del comité de experimentación humana responsable y de acuerdo con la Asociación Médica Mundial y la Declaración de Helsinki.

Confidencialidad de los datos: Los autores declaran que han seguido los protocolos de su centro de trabajo sobre la publicación de datos de pacientes.

Derecho a la privacidad y consentimiento informado: Los autores han obtenido el consentimiento informado de los pacientes y/o sujetos referidos en el artículo. Este documento obra en poder del autor de correspondencia.

\section{Conflicto de intereses}

Los autores declaran no tener conflicto de intereses. 


\section{Referencias}

1. Berfelo FJ, Kersbergen KJ, van Ommen $\mathrm{CH}$, et al. Neonatal cerebral sinovenous thrombosis from symptom to outcome. Stroke 2010;41:1382-8.

2. Fitzgerald KC, Williams LS, Garg BP, Carvalho KS, Golomb MR. Cerebral sinovenous thrombosis in the neonate. Arch neurol. 2006;63:405-9.

3. Heller C, Heinecke A, Junker R, et al. Cerebral venous thrombosis in children: a multifactorial origin. Circulation. 2003;108(11):1362-7.

4. Garrido-Barbero M, Arnaez J, Loureiro B, Arca G, Agut T, García-Alix A. The Role of Factor V Leiden, Prothrombin G20210A, and MTHFR C677T Mutations in Neonatal Cerebral Sinovenous Thrombosis. Clin Appl Thromb Hemost. 2019;25:1076029619834352. doi: $10.1177 / 1076029619834352$. PubMed PMID: 31025572.

5. Lolli V, Molinari F, Pruvo JP, Soto Ares G. Radiological and clinical features of cerebral sinovenous thrombosis in newborns and older children. J Neuroradiol. 2016;43:280-9.

6. Fitzgerald KC, Williams LS, Garg BP, Carvalho KS, Golomb MR. Cerebral sinovenous thrombosis in the neonate. Arch Neurol. 2006;63:405 9.

7. Kersbergen KJ, Groenendaal F, Benders MJ, de Vries LS. Neonatal cerebral sinovenous thrombosis: neuroimaging and long-term follow-up. J Child Neurol. 2011;26:1111-20.

8. Agut T, Roca P, García-Alix A. [Neonatal status epilepticus undetected with single-channel aEEG]. An Pediatr (Barc). 2017;87:57-8.

9. Park WC, Chang JH. Clinical Implications of Methylenetetrahydrofolate Reductase Mutations and Plasma Homocysteine Levels in Patients with Thromboembolic Occlusion. Vasc Specialist Int. 2014;30:113-9.

10. Turan Ö, Anuk-İnce D, Olcay L, Sezer T, Gülleroğlu K, Yılmaz-Çelik Z, Ecevit A. Neonatal cerebral sinovenous thrombosis: Two cases, two different gen polymorphisms and risk factors. Turk J Pediatr. 2017;59:71-75.

11. Monagle P, Chan AKC, Goldenberg $\mathrm{NA}$, et al. Antithrombotic therapy in neonates and children: Antithrombotic Therapy and Prevention of Thrombosis, 9th ed: American College of Chest Physicians Evidence-Based Clinical Practice Guidelines. Chest. 2012;141(2 Suppl):e737S-e801S.

12. Roach ES, Golomb MR, Adams R, et al. Management of stroke in infants and children: a scientific statement from a Special Writing Group of the American Heart Association Stroke Council and the Council on Cardiovascular Disease in the Young. Stroke. 2008;39:2644-91.

13. Rossor T, Arichi T, Bhate S, Hart AR, Raman Singh R. Anticoagulation in the management of neonatal cerebral sinovenous thrombosis: a systematic review and meta-analysis. Dev Med Child Neurol. 2018;60:884-91. 